University of Nebraska - Lincoln

DigitalCommons@University of Nebraska - Lincoln

December 1987

\title{
Comment on "Relations between microscopic and macroscopic lowest-order optical nonlinearities of molecular crystals with one- or two-dimensional units"
}

\author{
K.M. White \\ University of Nebraska - Lincoln \\ Craig J. Eckhardt \\ University of Nebraska - Lincoln, ceckhardt1@unl.edu
}

Follow this and additional works at: https://digitalcommons.unl.edu/chemistryeckhardt

Part of the Chemistry Commons

White, K.M. and Eckhardt, Craig J., "Comment on "Relations between microscopic and macroscopic lowest-order optical nonlinearities of molecular crystals with one- or two-dimensional units"' (1987). Craig J. Eckhardt Publications. 25.

https://digitalcommons.unl.edu/chemistryeckhardt/25

This Article is brought to you for free and open access by the Published Research - Department of Chemistry at DigitalCommons@University of Nebraska - Lincoln. It has been accepted for inclusion in Craig J. Eckhardt Publications by an authorized administrator of DigitalCommons@University of Nebraska - Lincoln. 


\title{
Comments
}

Comments are short papers which comment on papers of other authors previously published in the Physical Review. Each Comment should state clearly to which paper it refers and must be accompanied by a brief abstract. The same publication schedule as for regular articles is followed, and page proofs are sent to authors.

\section{Comment on "Relations between microscopic and macroscopic lowest-order optical nonlinearities of molecular crystals with one- or two-dimensional units"}

\author{
K. M. White and C. J. Eckhardt \\ Department of Chemistry, University of Nebraska-Lincoln, Lincoln, Nebraska 68588-0304
}

(Received 1 June 1987)

An algebraic error in an earlier paper [J. Zyss and J. L. Oudar, Phys. Rev. A 26, 2028 (1982)] led to an interpretation regarding the desirable polarizability characteristics of a molecule for second-harmonic generation in crystals which is opposite to that actually desired. Optimum response is obtained from crystals comprised of molecules with significant in-plane polarizabilities.

In a fundamental theoretical study by Zyss and Oudar, ${ }^{1}$ expressions that relate the lowest-order macroscopic optical nonlinearities of molecular crystals to the oneor two-dimensional hyperpolarizabilities of the molecular entities themselves were derived for all applicable crystal point groups. Using these relationships, it was shown for the one-dimensional systems that specific orientations of the intramolecular charge-transfer axes in the crystals will optimize the macroscopic nonlinear coefficients $\left(d_{I J K}\right)$. As a part of their study, Zyss and Oudar determined those orientations for the $d_{I J K}$ coefficients involved in collinear phase-matched secondharmonic generation (SHG). They also pointed out that the efficiency of the SHG process would be increased should it be achieved under noncritical phase-matching (NCPM) conditions.

The requirements for NCPM, in which the collinear phase-matched propagation direction coincides with a principal dielectric axis of the crystal, were derived in terms of molecular polarizabilities for a biaxial crystal composed of molecules having one-dimensional hyperpolarizabilities and belonging to the point group 2. Examination of the derivations, however, reveals that correction of a sign error discovered in the result significantly alters the interpretation given in the ensuing discussion. Since the information available from this work has great potential as a blueprint in the development and preparation of organic molecular crystals that are capable of more efficient SHG, it is important that corrections be noted and conclusions based on the new result provided.

For the purpose of the derivation, the axial system employed by Zyss and Oudar (Fig. 4 of Ref. 1) is used. The $y$ axis of the molecule is chosen to be in the direction of the one-dimensional molecular hyperpolarizability $\left(\beta_{u u u}\right)$ and is oriented as shown in Fig. 1 , where $\theta$ is the angle between the molecular $y$ axis and the crystal symmetry axis ( $Y$ axis) of the point group 2 . The crystal $X$ and $Z$ axes are defined so that the $y$ axis lies in the $Y Z$ plane. The optimal phase-matchable nonlinear coefficient in this system is $d_{Y Z Z}$, which is given by

$$
d_{Y Z Z}=N \beta_{u u u} \cos \theta \sin ^{2} \theta \text {, }
$$

where $N$ is a factor that accounts for local fields and the number of molecules per unit volume in the crystal. The value of $d_{Y Z Z}$ is at a maximum when $\theta=54.74^{\circ}$.

The most efficient use of $d_{Y Z Z}$ for collinear phasematched SHG will occur only if the $X$ axis is a phasematchable direction. Then, for the case of type-I phase matching, light propagating along the $X$ axis with its electric field polarized along the $Z$ axis will interact with the crystal second-order susceptibility, thereby giving rise to frequency-doubled light polarized along the $Y$ axis. On the other hand, if the phase-matchable direction is not along the crystal $X$ axis, the effective value of $d_{Y Z Z}$ will be reduced by angular factors dependent upon the projections of the electric field vectors of the fundamental and harmonic waves onto the $Z$ and $Y$ axes, respectively. Moreover, if the phase-matchable direction for either of these cases does not coincide with a principal dielectric axis in the crystal, the divergence of a focused fundamental beam and its "walk-off" from the harmonic beam will impose additional limits on the efficiency of the SHG process. ${ }^{2,3}$

The objective is to determine what conditions must exist in the crystal so that the $X$ axis (Fig. 1) will not only be a phase-matchable direction for the frequency of interest, but will also be nearly coincident with one of the principal dielectric axes of the medium. Taking a molecule having a highly one-dimensional hyperpolarizability 


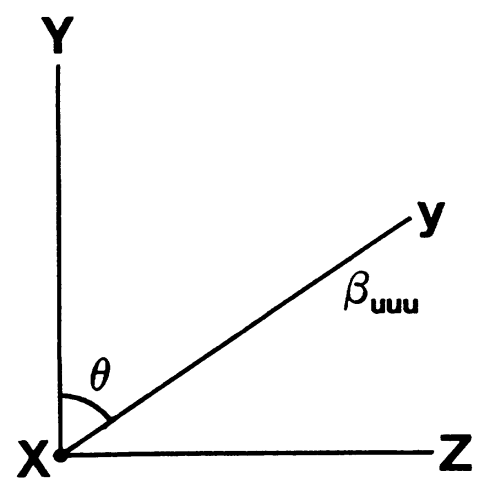

FIG. 1. Axial system for a crystal belonging to point group 2 that is composed of molecules having a one-dimensional hyperpolarizability $\beta_{u u u}$. The $Y$ axis is the crystal symmetry axis and the $Y Z$ plane is chosen to contain the molecular $y$ axis, which is taken to coincide with $\beta_{\text {uиu }}$.

and a linear polarizability such that $\alpha_{X X}^{\prime}=\alpha_{Y Y}=\alpha_{\|}$and $\alpha_{Z Z}=\alpha_{\perp}$, Zyss and Oudar showed ${ }^{1,4}$ that if the mean plane $(x y)$ of the molecule is perpendicular to the crystal $Y Z$ plane, then the molecular $x$ axis and crystal $X$ axis will coincide with a principal dielectric axis of the crystal. The phase-matching condition, in terms of crystal refractive indexes, requires that

$$
n_{Y}^{2 \omega}=n_{Z}^{\omega}
$$

This was shown to lead to

$$
\alpha_{Y Y}^{2 \omega}=\alpha_{Z Z}^{\omega},
$$

where, in general, $\alpha_{I I}^{\omega}$ is the polarizability of the molecule at a frequency $\omega$ using the $X-Y-Z$-crystal coordinates. Writing this in terms of $\alpha_{\|}$and $\alpha_{1}$, one obtains

$$
\alpha_{\|}^{2 \omega} \cos ^{2} \theta+\alpha_{\perp}^{2 \omega} \sin ^{2} \theta=\alpha_{\|}^{\omega} \sin ^{2} \theta+\alpha_{\perp}^{\omega} \cos ^{2} \theta
$$

For the optimal orientation of the molecular hyperpolarizability, which occurs when $\theta=54.74^{\circ}$, this expression becomes

$$
2=\tan ^{2} \theta=\left(\alpha_{\|}^{2 \omega}-\alpha_{\perp}^{\omega}\right) /\left(\alpha_{\|}^{\omega}-\alpha_{\perp}^{2 \omega}\right) .
$$

Since this result is opposite in sign to the one obtained earlier [Eq. (4.12) in Ref. 1], it leads to a new set of conclusions that are contrary to those advanced previously. Firstly, it is now seen that the relation can be satisfied by a molecule in which $\alpha_{\|}^{\omega} \gg \alpha_{1}^{\omega}$. This is indeed fortunate since a majority of the organic molecules that have been studied for their second-order nonlinearities are composed of benzenoid rings, in which the out-of-plane polarizability is significantly less than the in-plane component. Secondly, there is no longer the need for derivatization to coarsely increase the amount of out-ofplane molecular polarizability to satisfy Eq. (5). Consequently, the problems that could arise from the presence of additional groups in the molecule, such as a change in orientation of the molecule from a previously optimized position, would then be avoided. Finally, small alterations in the molecule, such as the introduction of a heteroatom into the benzene ring, may have a pronounced effect on the above relation without significantly altering the crystal structure of the material. This opens the way for testing of these theoretical conditions and is a step closer to the engineering of organic crystals that will have optimal SHG efficiencies.

This work was supported by a grant from 3M Company.

ley, New York, 1973).

${ }^{4}$ J. L. Oudar and J. Zyss, Phys. Rev. A 26, 2016 (1982).

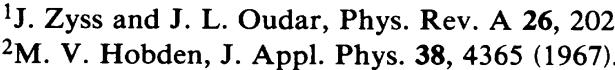

${ }^{3}$ F. Zernike and J. E. Midwinter, Applied Nonlinear Optics (Wi- 